\title{
Obstacle detection and detour system using line laser and camera
}

\author{
Miki Suetsugu, Shiyuan Yang, Seiichi Serikawa \\ Department of Electrical and Electronic Engineering, Kyushu Institute of Technology, Kitakyushu, Japan \\ *Corresponding Author: suetsugu.miki800@mail.kyutech.jp
}

\begin{abstract}
This paper develops a system that can easily and widely detect and avoid obstacles with one system by using a line laser and a camera. In order to use the automatic guided vehicle safely indoors, it is necessary to detect obstacles and avoid collisions. Therefore, we installed a line laser and a camera on the mobile robot to verify the accuracy of the system that detects and avoids obstacles. Conventional automatic guided vehicles include a method of detecting using a plurality of sensors and a method of detecting using LiDAR. However, these have problems that they are easily affected by disturbance and that the sensor itself is expensive and impractical. In this study, by using a line laser and a camera, it is possible to detect easily and widely with one system.
\end{abstract}

Keywords: Electronic, Robot, Sensor, Camera, Laser, Image processing, Camera, Measurement.

\section{Introduction}

In recent years, automated guided vehicles have been used in many fields, including production lines for automobiles and food, and for transporting equipment in hospitals. The global market, including companies that provide automated guided vehicles and related technologies, is expected to grow to $\$ 4.3$ billion by 2027 . [1] The current automatic guided vehicle will stop if there is an obstacle on the track. In addition, there is a risk of collision with obstacles. Therefore, it is necessary to detect obstacles and avoid them appropriately. Conventional obstacle detection systems include ultrasonic sensors, PSD sensors, cameras, and LiDAR. However, these have some drawbacks. Ultrasonic sensors and PSD sensors can only measure in one dimension. [2][3] A large number is required for wide-range measurement. In addition, an error occurs due to the influence of ambient noise and reflected light. The method using a camera detects obstacles from the difference between the preset background image and the input image. [4][5] However, this method requires updating the background image and takes a long time to process. LiDAR is widely used in the field of autonomous driving [6]. However, this has the disadvantages that the sensor itself is expensive and difficult to introduce, and that measurement cannot be performed if the laser is not reflected.

Therefore, the purpose of this research is to develop a system that easily and clearly detects and avoids obstacles using a line laser and a camera.

\section{Methods}

\subsection{Obstacle detection}

The system configuration diagram is shown in Fig. 1. As shown in Fig. 1, the floor is irradiated with a green line laser and the state is input by the camera. As shown in Fig. 2 front view, when there is an obstacle in front of the robot, the line laser is not straight but interrupted.

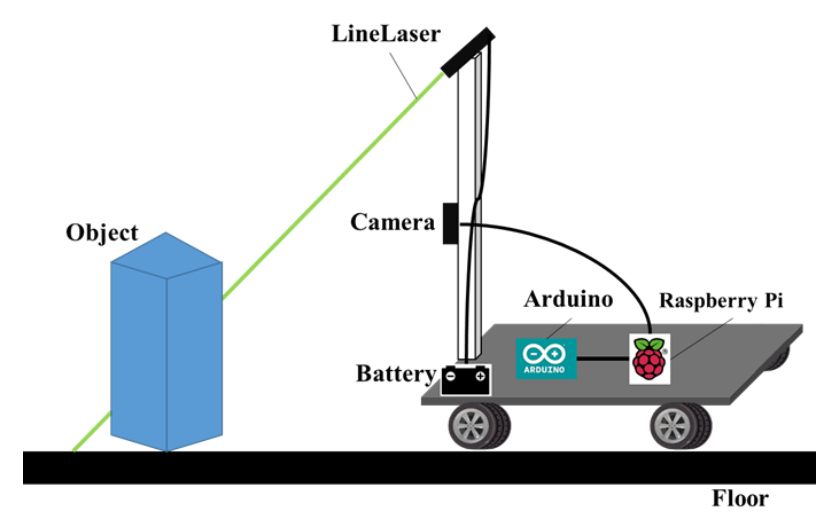

Fig. 1. Side view 


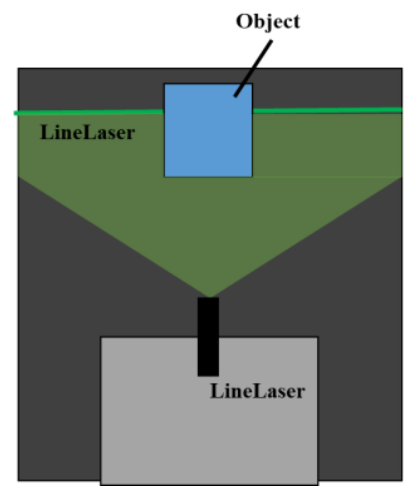

Fig. 2. Front view

Only the line laser is extracted from the image input from the camera. Fig. 3 shows an image of only the line laser extracted.

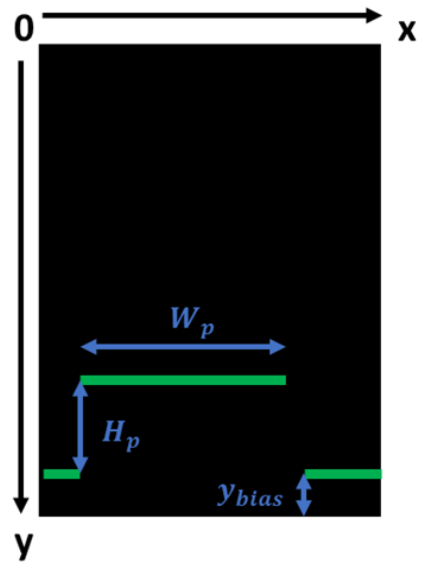

Fig. 3. Image after line laser extraction

From Fig. 3, characteristic changes can be seen in the area where the obstacle exists, depending on the shape of the obstacle. Using this change, the presence or absence of obstacles is detected and the height and width of the obstacles and the distance to the obstacles are calculated. The image takes the $\mathrm{x}$-axis in the horizontal direction and the $y$-axis in the vertical direction with the upper left as the origin, and converts it to the actual length based on the number of pixels $H_{p}$ and $W_{p}$ when there is an obstacle. $y_{\text {bias }}$ [pixel] is defined as the height to the position of the line laser beam radiated to the floor of the image. Fig. 4 shows the positional relationship with the camera, line laser, and obstacles. The point $\mathrm{P}$ is the irradiation position of the line laser, and the blue area is the shooting range of the camera.

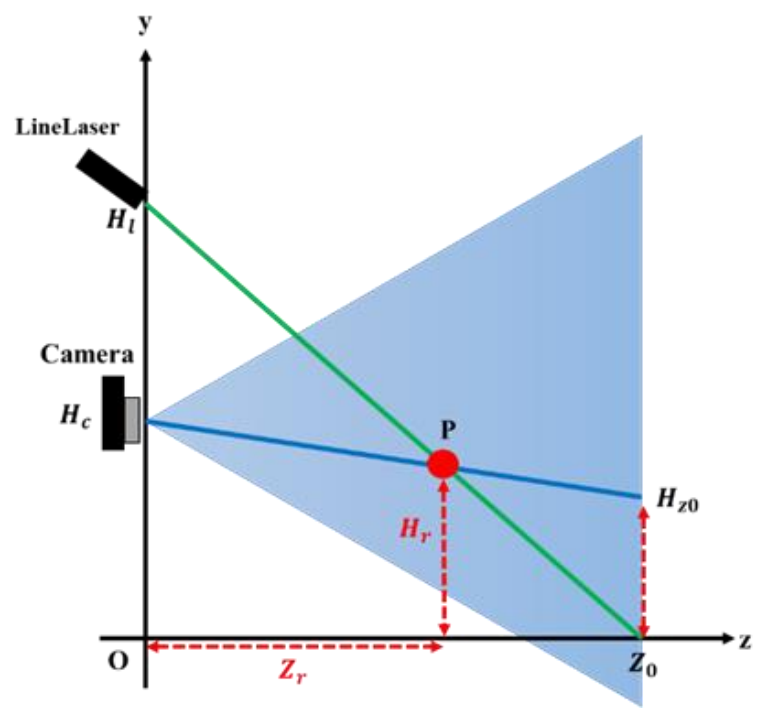

Fig. 4. Positional relationship of measurement system

The height of the obstacle $H_{r}$ [cm] can be obtained from $H_{p}$, and the distance $Z_{r}[\mathrm{~cm}]$ between the mobile robot and the obstacle can be calculated by Eq. (1).

$$
Z_{r}=\frac{Z_{0}\left(H_{l}-H_{c}\right)}{H_{z 0}-H_{c}+H_{l}}
$$

Using this, the width of the obstacle $W_{r}$ [cm] can be calculated by Eq. (2). Let the horizontal resolution in Fig. 3 be $P_{w}$ and the focal length of the camera $f$.[7]

$$
W_{r}=\frac{Z_{r} W_{i} W_{p}}{f P_{w}}
$$

\subsection{Obstacle detour}

After detecting obstacles, driving efficiency can be improved by avoiding them and running without stopping. If there is an obstacle at the position shown in Fig. 5, the mobile robot determines that the optimum avoidance direction is "left". Let $x_{r}$ be the line segment connecting the edge of the obstacle and the point at the distance $Z_{r}$ on the extension of the width of the robot.

Step1 The Obstacle detour angle $\theta\left[{ }^{\circ}\right]$ is as shown in Eq. (3). The angle $\theta$ can be obtained from the right triangle of the distances $x_{r}, Z_{r}$.

$$
\theta=\tan ^{-1} \frac{x_{r}}{Z_{r}}
$$

Step2 The distance $D_{r}$ can be calculated by the threesquare theorem. The distance $D_{r}[\mathrm{~cm}]$ is calculated by Eq. (4).

$$
D_{r}=\sqrt[2]{x_{r}^{2}+Z_{r}^{2}}
$$




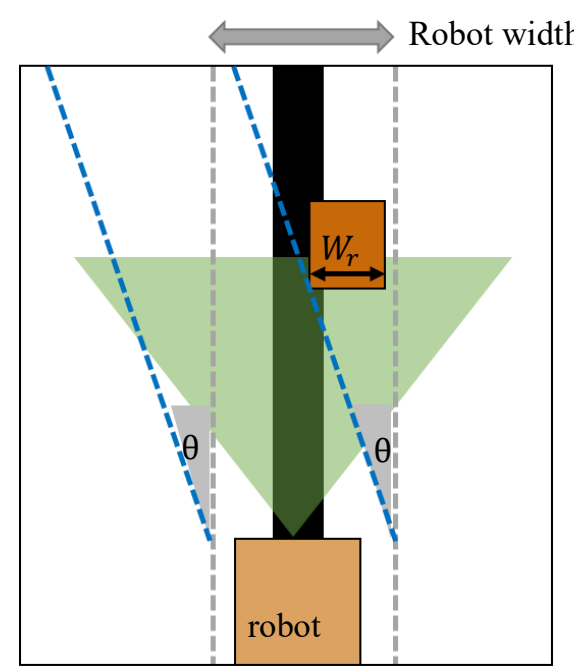

Step 1

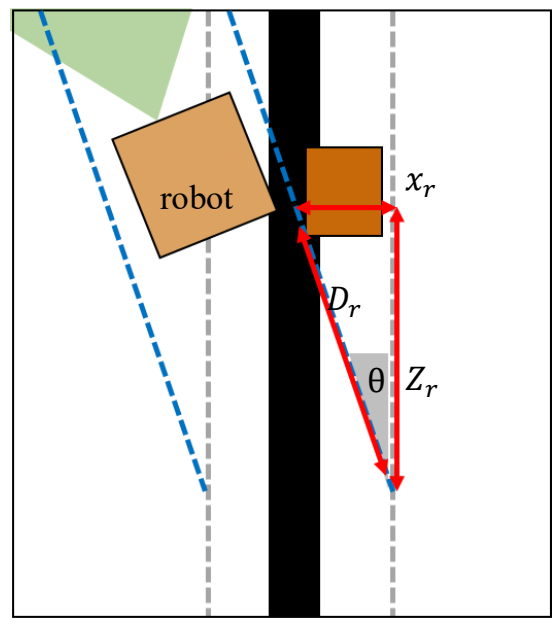

Step2

Fig. 5. Method of obstacle avoidance

\section{Experiment and discussion}

\subsection{Obstacle detection}

The distance $Z_{0}$ between the line laser irradiation destination and the robot was set to $80[\mathrm{~cm}]$. The robot continued to acquire data in real time and verified the obstacle detection accuracy. The robot advances at a speed of $14.3[\mathrm{~cm} / \mathrm{s}]$. Place an obstacle $100[\mathrm{~cm}]$ ahead of the robot. Detect an obstacle and measure the width $W_{r}$ of the obstacle and the distance $Z_{r}$ to the obstacle. Go straight until $Z_{r}=50[\mathrm{~cm}]$ and measure every $1[\mathrm{~cm}]$. In the experiment, a box with a width of $15.5[\mathrm{~cm}]$ was used as an obstacle. Image of the experiment is shown in Fig. 6.

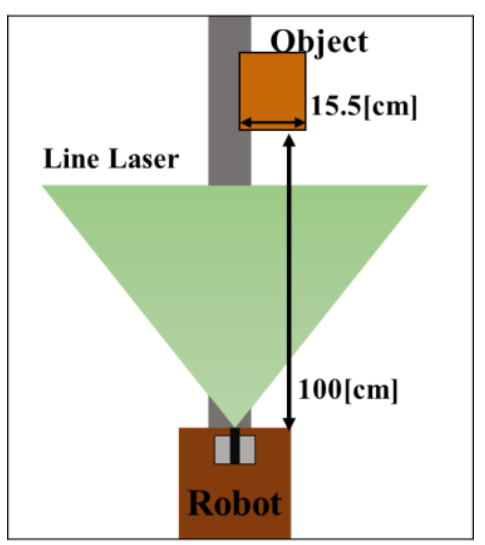

Fig.6. Image of experiment

The state of obstacle detection is shown in Fig. 7. The experimental results are shown in Fig. 8. From Fig. 8, the obstacle could be detected from the distance $Z_{r}=79[\mathrm{~cm}]$ between the obstacle and the mobile robot. This factor is based on the principle of detecting obstacles. Since obstacles are detected by the deviation of the line laser, obstacles cannot be detected when the irradiation distance of the line laser is $Z_{0}=Z_{r}$.

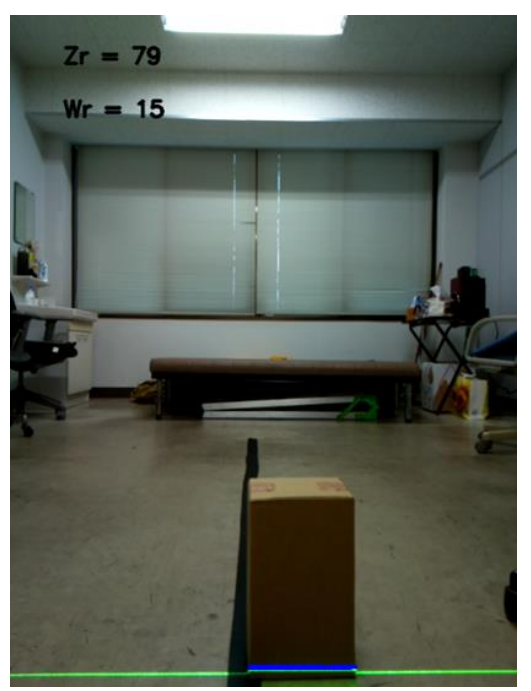

Fig. 7. Actual state of obstacle detection

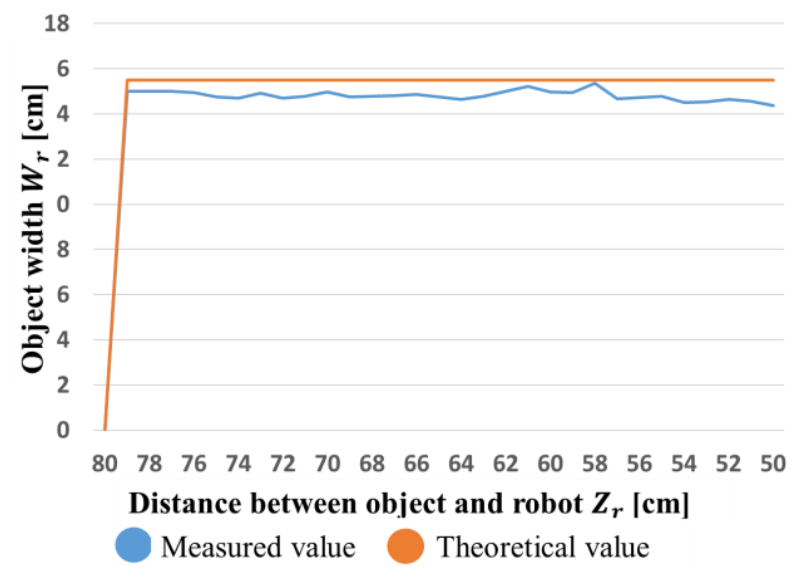

Fig. 8. Obstacle detection result 
From Fig. 8, it was possible to measure accurately without a large error. The maximum error was 1.12 [cm], the minimum error was $0.15[\mathrm{~cm}]$, and the average error was $0.69[\mathrm{~cm}]$. The average error was less than $1 \mathrm{~cm}$, and it is considered that the detection was accurate. It is considered to be a practical obstacle detection system that can measure in real time.

\subsection{Obstacle detour}

The obstacle avoidance system proposed in this study finds the obstacle avoidance angle $\theta$ by using the obstacle width $W_{r}$ obtained in $\mathbf{3 . 1}$ and the distance $Z_{r}$ between the robot and the obstacle. The distance $Z_{0}$ between the line laser irradiation destination and the robot was set to 80 [cm]. The mobile robot moves at a speed of 14.3 [cm / s]. After finding the obstacle avoidable angle $\theta$, the mobile robot changes its angle direction and advances the distance $D_{r}$ to detour the obstacle. Fig. 8 shows how to detour obstacles.

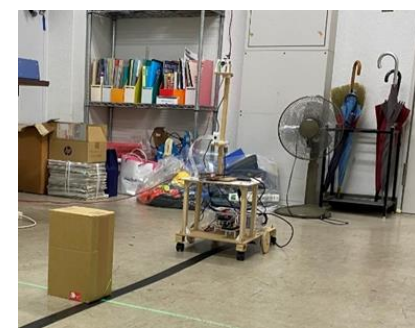

Step.1

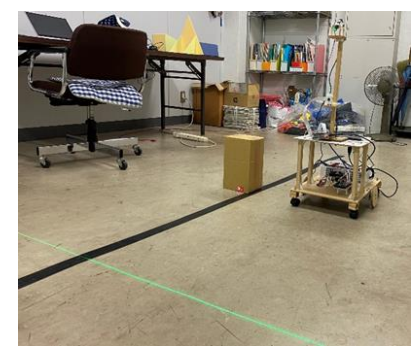

Step.3

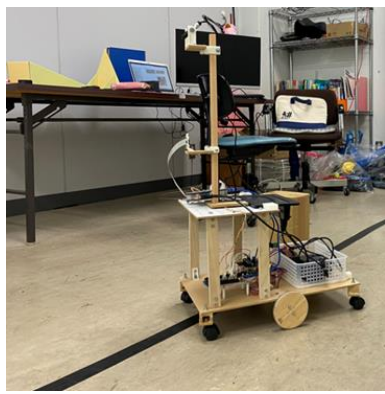

Step.5

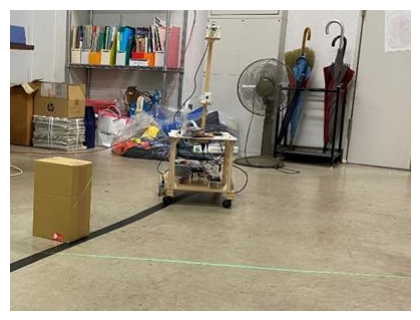

Step. 2

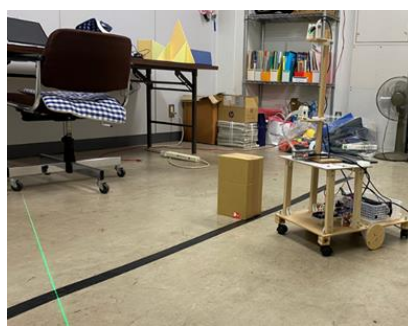

Step. 4

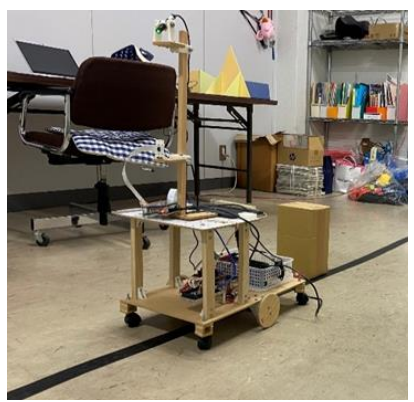

Step.6
Fig. 8 Obstacle detour
From Fig. 8, the mobile robot can move while detecting obstacles and avoiding them. After starting the mobile robot, it went straight along the line and detected an obstacle (Step.1). If you continue to go straight on the line, you will collide, so you changed your course to the left, where the movable area is wide (Step.2). At this time, since the width $W_{r}$ of the obstacle and the distance $Z_{r}$ between the obstacle and the mobile robot are known, the avoidable angle $\theta$ was obtained and the direction was changed. After the direction was changed, the distance $D_{r}$ was advanced because there were no obstacles within the range of the mobile robot (Step.3). After traveling the distance $D_{r}$, the direction was changed by $-2 \theta$ to avoid obstacles (Step.4). After changing direction, the mobile robot went straight (Step.5) and went straight on the black line, which is the running path (Step.6).

From Fig. 8, the mobile robot can detect obstacles in real time and avoid them without collision, and good results are obtained. It is considered that practical obstacle avoidance is possible by integrating the distance information and the width information of the obstacle.

\section{Conclusions}

Currently, the demand for indoor mobile robots is on the rise. Therefore, a system for detecting indoor walls is indispensable, and a highly accurate system is required. In the conventional method, a plurality of sensors are incorporated or an expensive sensor is used. However, this research system consists of a line laser and a camera. A lowcost, high-precision system was realized. Many mobile robots use basic cameras. This system can be implemented simply by installing a line laser on top of the mobile robot.

In this research, in order to allow the mobile robot to avoid obstacles, avoid collisions, and run without stopping, an approach using a line laser and a camera, which is different from the conventional obstacle detection method, is used for obstacles.

The method proposed in this study depends on the irradiated part of the line laser. The part not irradiated with the line laser cannot be detected. Improving this point is a future task. It is thought that a wider range of obstacles can be detected by using a camera with a wide angle and low distortion. In addition, in this system, experiments were conducted using one line laser, but it is possible to reduce the undetectable part by increasing the number of lasers with different irradiation angles. 


\section{References}

(1) LNEWS: "IDC Japan / Autonomous mobile robots will reach a market size of 56.1 billion yen in 2023"https://www.lnews.jp/2019/05/10514309.html, 2019

(2) Hidenori Ishihara, Toshio Fukuda, Kazuhiro Kosuge and F.Arai : "An approach to autonomous microrobot micro line trace robot with reflex algorithm", IEEE /ASME Transactions, Vol. 1, No. 1, pp.68-79, 1996

(3) Jeong-Gi Lee, D.Kim, Jin-Young Suk: "Development of a 3D Position Determination Device using PSD sensor", IEEE, pp.1733-1736, 2010

(4) Oe, Motoko, Tomokazu Sato, and Naokazu Yokoya : "Estimating camera position and posture by using feature landmark database.”, Scandinavian Conference on Image Analysis. Springer Berlin Heidelberg, pp.171181,2005

(5) Han Wang, Zhuo Wei, Sisong Wang, Chek Seng Ow, Kah Tong Ho and Benjamin Feng: "A vision-based obstacle detection system for Unmanned Surface Vehicle", IEEE, 5, pp.364-369, 2011

(6) Cristiano Premebida, Goncalo Monteiro, Urbano Nunes, Paulo Peixoto : "A Lidar and Vision-based Approach for Pedestrian and Vehicle Detection and Tracking", IEEE, , pp.1044-1049, 2007 